\title{
Myocardial-coronary fusion imaging with positron emission tomography and computed tomography: Benchmarking and slingshotting
}

\author{
Francesco Nudi, MD, ${ }^{\text {a,b,c }}$ Giuseppe Biondi-Zoccai, MD, MStat, ${ }^{\text {d,e }}$ Natale Di \\ Belardino, MD, ${ }^{f}$ Alessandro Nudi, $M D,{ }^{a}$ and Orazio Schillaci, $M D^{\mathrm{e}, \mathrm{g}}$ \\ a Service of Hybrid Cardiac Imaging, Madonna della Fiducia Clinic, Rome, Italy \\ b Ostia Radiologica, Rome, Italy \\ c Replycare, Rome, Italy \\ d Department of Medico-Surgical Sciences and Biotechnologies, Sapienza University of Rome, \\ Latina, Italy \\ e IRCCS Neuromed, Pozzilli, Italy \\ f Division of Cardiology, Anzio-Nettuno Hospital, Anzio, Italy \\ g Department of Nuclear Medicine, Tor Vergata University of Rome, Rome, Italy
}

Received Nov 12, 2018; accepted Nov 12, 2018

doi: $10.1007 / \mathrm{s} 12350-018-01538-2$

\section{See related article, pp. 1774-1784}

If there is knowledge, it lies in the fusion of the book and the street Louis "'Studs"' Terkel

The modern approach to coronary artery disease (CAD) and ischemic heart disease (IHD) has finally overcome the sickly dispute on the comparative diagnostic and prognostic accuracy of anatomic and functional non-invasive testing. ${ }^{1,2}$ Indeed, hybrid anatomo-functional non-invasive imaging can surpass the limitations inherent to each test alone, providing accurate details for diagnosis, prognosis, and management at the patient, vessel, and lesion level. ${ }^{1,3}$ Most importantly, hybrid imaging offers expanding opportunities to improve patient clustering and treatment, given that integrating details can also be obtained with single or multimodality imaging on myocardial metabolism (important to distinguish between viability and necrosis), systolic function

\footnotetext{
Funding This work was supported by Replycare, Rome, Italy.

Reprint requests: Francesco Nudi, MD, Service of Hybrid Cardiac Imaging, Madonna della Fiducia Clinic, Via Giuseppe Mantellini 3, 00179 Rome, Italy; francesco.nudi@gmail.com

J Nucl Cardiol 2020;27:1770-3.

$1071-3581 / \$ 34.00$

Copyright (c) 2018 American Society of Nuclear Cardiology.
}

(key to distinguish stunning from hibernation over time), diastolic function, ventricular geometry (to disentangle the impact of CAD on heart failure from heart failure progression per se), and dyssynchronization, as well as innervation/dysinnervation. ${ }^{1,4}$

Notably, hybrid imaging appears "beneficial irrespective of its core components," which may include, on top of computed tomography angiography (CTA), any of the latter alone or in combination: stress echocardiography, single-photon emission-computed tomography (SPECT) with myocardial perfusion imaging (MPI), positron emission tomography (PET) with MPI, cardiac magnetic resonance (CMR), computed tomography myocardial perfusion (CTP), and computed tomography fractional flow reserve (CTFFR), PET, and SPECT with assessment of coronary flow reserve (CFR). ${ }^{2}$ Accordingly, institutional and operator expertise, with the complex interplay between training, skill, and volume, may impact on the choice and performance of each component of hybrid imaging.

Yet, for many reasons PET can be considered as one of the best players in this diagnostic dynamic duo. ${ }^{3}$ Specifically, PET can provide detailed qualitative, semiquantitative, and quantitative imaging using different radionuclides such as ${ }^{82} \mathrm{Rb},{ }^{13} \mathrm{~N}$-ammonia, ${ }^{15} \mathrm{O}$ water, and ${ }^{18} \mathrm{~F}$-fluoride, with heightened spatio-temporal resolution. ${ }^{5,6}$ Indeed, the capability of PET to provide accurate measurements for myocardial blood flow (MBF), myocardial flow reserve (MFR), and relative flow reserve (RFR), a nuclear cardiology proxy of 
fractional flow reserve (FFR), remains unparalleled, offering high diagnostic accuracy, decreased radiation exposure, and the unique ability to quantify non-invasively rest and stress $\mathrm{MBF}$ in absolute terms, i.e., in $\mathrm{mL}$ per minute per gram of myocardial tissue. ${ }^{5}$

It is also important for nuclear cardiologists to remember that invasive FFR, currently considered by many the gold standard for the functional assessment of $\mathrm{CAD}$, is only a relative measurement of flow reserve, and was initially validated using absolute and relative perfusion measurements obtained with PET. ${ }^{7,8}$ The possibility to measure coronary flow is considered by some the key Achilles' heel of this and similar tests, at least in the absence of anatomic details, as it heavily depends on microvascular dysfunction. For instance, chronic cigarette smoking is per se associated with a significant, albeit reversible after cessation, reduction in CFR. $^{6}$

In recent years, FFR has emerged over the years as a gold standard for the diagnostic, prognostic, and therapeutic approach to patients with stable CAD, thanks to its independent and almost invariant precision. Indeed, it only depends on luminal area, lesion length, and the subtending viable myocardium, as proven by several landmark studies on the diagnostic and prognostic accuracy of FFR, as well as by dedicated randomized trials. $^{7-9}$ Yet, even FFR cannot be considered perfect, but rather as an "imperfect reference standard for myocardial ischemia,' as it is invasive, entailing radiation exposure, potentially associated with procedural complications, unreliable in case of spasm, dissection, or thrombus, and unfeasible in case of total occlusion. ${ }^{8}$ In addition, the fact that it is performed by definition after invasive coronary angiography limits its scope and gatekeeping potential.

The typical approach followed so far for the integration of CTA and functional testing, including PET, has been to provide hybrid images detailing the coronary arteries superimposed on color-coded ventricular surface. $^{2}$ This approach offers the opportunity for visual integration but it requires interpretative skills and does not overcome the limitations inherent to the visual analysis of relative perfusion patterns. In addition, integrating scoring systems for coronary anatomy (e.g., the Gensini, Coronary Artery Surgery Study [CASS], or Synergy between PCI with Taxus and Cardiac Surgery [SYNTAX] ones), and for functional assessment (e.g., quantitative perfusion score [QPS], maximal ischemia score [MIS], or vessel-related ischemia [VRI] score) have proved so far quite difficult, except for the unique scenario of CTFFR, which integrates by default anatomic and functional imaging. ${ }^{10-12}$ Yet, the concept of comparing non-invasively anatomic and functional scores of CAD burden remains very appealing. ${ }^{2,13,14}$
This issue of the Journal provides an important contribution to the field of hybrid anatomo-functional non-invasive imaging for CAD and IHD by Piccinelli et al. ${ }^{15}$ The authors build upon extensive experience with both CT and PET, and capitalize on decade long efforts at fusion of anatomic and functional details. Most interesting, they revisit two key pathophysiologic underpinnings of PET, i.e., the choice of the region of interest (ROI) for arterial input to measure absolute perfusion, and the potential exploitation of basal to apical gradients of MBF, CFR, and RFR for the differential diagnosis between focal epicardial CAD, diffuse epicardial CAD, and microvascular dysfunction. ${ }^{16}$ Indeed, focal CAD is typically associated with abnormal gradients, whereas diffuse CAD and microvascular dysfunction do not. ${ }^{17-19}$ The shared expectation is that including basal to apical gradients will improve per patient, per vessel, and per lesion accuracy in diagnosis and prognosis, thus substantially refining decision making. Accordingly, the basic rationale of their approach is associating a specific myocardial segment to a specific coronary segment, using CTA for actual anatomic mapping, and then comparing results of PET in that specific volume of interest during stress-rest. This amounts to appealing and succinct images in which coronary arteries are color-coded according to PET data, thus resembling a tri-dimensional reconstruction of three-vessel FFR pullbacks, similarly to CTFFR. ${ }^{13}$

This technological development was first applied by Piccinelli et al in a series of 16 apparently healthy subjects undergoing CTA and PET. They found that myocardial-coronary fusion imaging was possible and feasible in all cases as long as the most basal segments were flagged. In addition, they could generate benchmark pullback values for the whole heart, the left anterior descending (LAD), the left circumflex (LCX), and the posterior descending artery (PDA) of stress $\mathrm{MBF}$, rest MBF, MFR, and RFR. Comparative data were collected for the whole analysis set and the set obtained after excluding basal segments, and for women vs men. In addition, the technology was tested in a single case of a 46-year-old woman who underwent CTA, PET, and invasive coronary angiography with measurement of FFR and iFR. Notably, this patient had an anatomically and functionally significant lesion in the mid LAD, with an FFR of 0.73 (reference: $\geq 0.80$ ) and an iFR of 0.87 (reference: $\geq 0.90$ ). Accordingly, standard parametric analysis of perfusion patterns at PET showed a large reversible defect in the mid anterior and apical regions. Unfortunately, no details on the severity of the defect were provided, which is actually much more informative than size for diagnostic, prognostic, and management. Indeed, it is possible that this was not a severe defect, but a less than severe and impactful one. 
As hoped, coronary-myocardial fusion imaging showed a substantial decrease in RFR along the mid portion of the reconstructed LAD. However, all values obtained from fusion imaging, including stress MBF, MFR, and RFR appeared above the lowest benchmarking limits. Yet, processed color-coded coronary images exhibited a good agreement with FFR at eyeballing analysis, thus suggesting that if suitably refined, this methodology could indeed have substantial potential. Accordingly, we expect additional studies from these investigators and others to better refine their methodology, increase benchmarking data, and test in larger sets of patients their technology.

The contribution by Piccinelli and colleagues, while quite interesting and useful to guide further research and practice, has however several key limitations. First, the methods themselves are poorly defined from a technological perspective (e.g., there are no data on the imaging suites used or the software package employed for fusion). Second, the retrospective design, small sample size, and focus on apparently healthy subjects limit the precision and clinical validity of their results. Inclusion of cases from different continents, while boosting external validity, may have generated heterogeneity. The use of exercise stress in the future, relying on tracers with longer half-life, will possibly reduce the dispersion in normal values of RFR in comparison to adenosine (which was the stressor used by Piccinelli et al), by having the former stress a less-marked effect on the ability of increasing basal MBF. ${ }^{20}$ The potential advantage of exercise stress in comparison to pharmacologic stress to evaluate ischemia severity will also warrant further appraisal. Indeed, both ${ }^{15} \mathrm{O}$-water (which is considered by many the ideal PET tracer) and ${ }^{13} \mathrm{~N}$ ammonia require on-site cyclotron, whereas ${ }^{82} \mathrm{Rb}$ is much more common given that it is generator-produced. Accordingly, further appraisal of the proposed coronarymyocardial fusion imaging using ${ }^{15} \mathrm{O}$-water and ${ }^{82} \mathrm{Rb}$ is eagerly awaited. Moreover, enrolment of as many 3 (19\%) subjects with coronary calcium score $>400$, as well $4(25 \%)$ with diabetes mellitus and 11 (69\%) with hypertension, may have lead to the inclusion of cases with no angiographically obstructive coronary lesion (as demonstrated by CTA), but already significant plaque burden and most likely endothelial dysfunction.

More technically, the choice of analyzing only the region of myocardium that is directly subtended by an arbitrary 6-mm band under the anatomic vessel location is not based on any anatomic or pathophysiologic detail, and risks appearing purely subjective. In addition, the fact that the very patient with significant CAD at invasive assessment (anatomic and functional) chosen to anecdotally test the proposed reference ranges for MBF, MFR, and RFR yielded results which were not different from those obtained in apparently healthy subjects casts a shadow of doubt on the precision of the proposed approach. Accordingly, the possibility to exclude the basalmost segments to increase accuracy may potentially lead to an underestimation of functionally significant lesions located in the left main, proximal LAD, proximal LCX, proximal ramus intermedius, and the whole right coronary artery. Yet, the possibility of excluding such basal segments may reduce the risk of underestimating PET indices such as MBF and MFR. Even the concept of considering all vessels of similar length appears as an oversimplification, potentially leading to spuriously precise results. Finally, the potential for conflicts of interest should be borne in mind.

The main goals of cardiovascular imaging specialists are twofold: first, stratifying patients with negative tests toward the best management (from addressing risk factors to pharmacologic therapy more or less intense and inclusive of different drug agents); second, stratifying subjects with positive tests toward the choice between optimal medical therapy and revascularization, and in the latter group between percutaneous and surgical revascularization. Accordingly, the most interesting and incrementally valuable scenario from hybrid imaging occurs when the two tests disagree. The negativity of both tests, which is the rosiest occurrence for the patient, represents only one of the many possible combinations of test results, and, in particular, the very one in which there is less incremental value of the combined tests in comparison to the single tests alone.

In light of the totality of pathophysiologic and clinical evidence, including Piccinelli et al contribution, hybrid imaging continues to increase its importance in the diagnostic, prognostic, and therapeutic approach to patients with CAD. ${ }^{1}$ Yet, we should remain aware that simply generating esthetically appealing images (at risk of being spuriously precise or overly reductive) does not necessarily bring us toward the only truly beneficial integration, which is conceptual and best accomplished in the human mind. ${ }^{2}$ In addition, anatomic, functional, and hybrid assessment cannot disregard the ongoing quest for appropriate definition of the strengths and weaknesses of qualitative, semiquantitative, and quantitative assessment of imaging results. ${ }^{4}$ Finally, while PET has several unique features, it is expensive and its use in routine cardiovascular practice still a niche one. ${ }^{3}$ Given its wider availability and efficiency, SPECT continues to play an important role in functional imaging as well as in hybrid imaging. ${ }^{2}$ Most importantly, new-generation cadmium-zinc-telluride suites enable today CFR assessment with SPECT too, thus providing further opportunities for multidimensional assessment of patients with CAD. 
In conclusion, myocardial-coronary fusion imaging with PET and CTA represents a promising concept to move forward with the integration of anatomic and functional imaging for $\mathrm{CAD}$, beyond the simplistic and relatively uninformative approach of current hybrid imaging packages. Whether such fusion approach will actually prove useful in clinical research and practice remains uncertain though, and large comparative studies are required as benchmarks before this technology may slingshot into mainstream use.

\section{Author Contribution}

FN has conceived this work, drafted the manuscript, and finally approved it. GBZ, NDB, AN, and OS provided critical suggestions, participated in manuscript revision, and finally approved it.

\section{Disclosure}

All authors declare that they have no conflict of interest.

\section{References}

1. Giannopoulos AA, Gaemperli O. Hybrid imaging in ischemic heart disease. Rev Esp Cardiol (Engl Ed) 2018;71:382-90.

2. Nudi F, Biondi-Zoccai G. Cadmium-zinc-telluride myocardial perfusion imaging: The dream of a single test gets nearer. J Nucl Cardiol 2018;25:550-4.

3. Heller GV, Hendel RC, editors. Nuclear cardiology: Practical applications. New York: McGraw-Hill; 2017.

4. Biondi-Zoccai G, Versaci F, Iskandrian AE, Schillaci O, Nudi A, Frati G, Nudi F. Umbrella review and multivariate meta-analysis of diagnostic test accuracy studies on hybrid non-invasive imaging for coronary artery disease. J Nucl Cardiol 2018. https://doi.org/ 10.1007/s12350-018-01487-w.

5. Stuijfzand WJ, Uusitalo V, Kero T, Danad I, Rijnierse MT, Saraste A, Raijmakers PG, Lammertsma AA, Harms HJ, Heymans MW, Huisman MC, Marques KM, Kajander SA, Pietilä M, Sörensen J, van Royen N, Knuuti J, Knaapen P. Relative flow reserve derived from quantitative perfusion imaging may not outperform stress myocardial blood flow for identification of hemodynamically significant coronary artery disease. Circ Cardiovasc Imaging 2015;8:e002400.

6. Feher A, Sinusas AJ. Quantitative assessment of coronary microvascular function: Dynamic single-photon emission computed tomography, positron emission tomography, ultrasound, computed tomography, and magnetic resonance imaging. Circ Cardiovasc Imaging 2017; 10:e06427.

7. De Bruyne B, Baudhuin T, Melin JA, Pijls NH, Sys SU, Bol A, Paulus WJ, Heyndrickx GR, Wijns W. Coronary flow reserve calculated from pressure measurements in humans. Validation with positron emission tomography. Circulation 1994;89:1013-22.

8. Stegehuis VE, Wijntjens GW, Piek JJ, van de Hoef TP. Fractional flow reserve or coronary flow reserve for the assessment of myocardial perfusion : Implications of FFR as an imperfect reference standard for myocardial ischemia. Curr Cardiol Rep 2018;20:77.

9. Verardi R, Fioravanti F, Barbero U, Conrotto F, Omedè P, Montefusco A, Moretti C, D'Amico M, Rinaldi M, Escaned J, D'Ascenzo F. Network meta-analysis comparing iFR versus FFR versus coronary angiography to drive coronary revascularization. $\mathrm{J}$ Interv Cardiol 2018. https://doi.org/10.1111/joic.12551.

10. Şahan E, Şahan S, Karamanlığlu M, Gül M, Kalaycı S, Boyacı A, Dereağzı $\mathrm{F}$. The impact of the extent and severity of coronary artery disease on fractional flow reserve measurements. Eur Rev Med Pharmacol Sci 2016;20:3434-9.

11. Nudi F, Pinto A, Procaccini E, Neri G, Vetere M, Tomai F, Gaspardone A, Biondi-Zoccai G, Schillaci O. A novel clinically relevant segmentation method and corresponding maximal ischemia score to risk-stratify patients undergoing myocardial perfusion scintigraphy. J Nucl Cardiol 2014;21:807-18.

12. Nudi F, Schillaci O, Neri G, Pinto A, Procaccini E, Vetere M, Frati G, Tomai F, Biondi-Zoccai G. Prognostic impact of location and extent of vessel-related ischemia at myocardial perfusion scintigraphy in patients with or at risk for coronary artery disease. J Nucl Cardiol 2016;23:274-84.

13. Novara M, D'Ascenzo F, Gonella A, Bollati M, Biondi-Zoccai G, Moretti C, Omedè P, Sciuto F, Sheiban I, Gaita F. Changing of SYNTAX score performing fractional flow reserve in multivessel coronary artery disease. J Cardiovasc Med (Hagerstown) 2012;13:368-75.

14. Collet C, Onuma Y, Miyazaki Y, Morel MA, Serruys PW. Integration of non-invasive functional assessments with anatomical risk stratification in complex coronary artery disease: The noninvasive functional SYNTAX score. Cardiovasc Diagn Ther 2017;7:151-8

15. Piccinelli M, Cho S-G, Garcia EV, Alexanderson E, Lee JM, Cooke CD, Goyal N, Santos Sanchez M, Folks RD, Chen Z, Votaw J, Koo B-K, Bom H-S. Vessel-specific quantification of absolute myocardial blood flow, myocardial flow reserve and relative flow reserve by means of fused $13 \mathrm{NH} 3 \mathrm{PET}$ and CCTA: Ranges in a low-risk population and abnormality criteria. J Nucl Cardiol 2018. https://doi.org/10.1007/s12350-018-01472-3.

16. Gould KL, Nakagawa Y, Nakagawa K, Sdringola S, Hess MJ, Haynie M, Parker N, Mullani N, Kirkeeide R. Frequency and clinical implications of fluid dynamically significant diffuse coronary artery disease manifest as graded, longitudinal, base-toapex myocardial perfusion abnormalities by noninvasive positron emission tomography. Circulation 2000;101:1931-9.

17. Al-Mallah MH, Sitek A, Moore SC, Di Carli M, Dorbala S. Assessment of myocardial perfusion and function with PET and PET/CT. J Nucl Cardiol 2010;17:498-513.

18. Gould KL. Clinical cardiac PET using generator-produced Rb-82: A review. Cardiovasc Intervent Radiol 1989;12:245-51.

19. Al Badarin F, Aljizeeri A, Almasoudi F, Al-Mallah MH. Assessment of myocardial blood flow and coronary flow reserve with positron emission tomography in ischemic heart disease: Current state and future directions. Heart Fail Rev 2017;22:441-53.

20. Werner RA, Chen X, Rowe SP, Lapa C, Javadi MS, Higuchi T. Moving into the next era of PET myocardial perfusion imaging: Introduction of novel 18F-labeled tracers. Int J Cardiovasc Imaging 2018;5:4. https://doi.org/10.1007/s10554-018-1469-z. 